\title{
Unsupervised Rehabilitation: effects of Exercise Training over the Long Run
}

\author{
Japy Angelini Oliveira Filho, Ana Cristina Leal, Valter Correia Lima, \\ Dirceu Vieira Santos Filho, Bráulio Luna Filho
}

São Paulo, SP - Brazil

Objective - To assess the safety and efficacy of unsupervised rehabilitation (USR) in the long run in low-risk patients with coronary artery disease.

Methods - We carried out a retrospective study with 30 patients divided into: group I(GI) - 15 patients from private clinics undergoing unsupervised rehabilitation; group II (GII) - control group, 15 patients from ambulatory clinic basis, paired by age, sex, and clinical findings. GI was stimulated to exercise under indirect supervision (jogging, treadmill, and sports). GII received the usual clinical treatment.

Results - The pre- and postobservation values in GI were, respectively: $V O_{2}$ peak $(\mathrm{mL} / \mathrm{kg} / \mathrm{min}), 24 \pm 5$ and $31 \pm 9$; $V \mathrm{O}_{2}$ peak/peak HR: $0.18 \pm 0.05$ and $0.28 \pm 0.13$; peak dou-

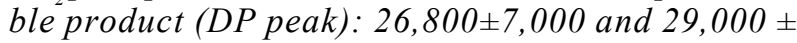
6,500; \% peak HR/predicted HRmax: $89.5 \pm 9$ and $89.3 \pm 9$. The pre- and post-values in GII were: $\mathrm{VO}_{2}$ peak $(\mathrm{mL} / \mathrm{kg} /$ min), $27 \pm 7$ and $28 \pm 5$; $V O$ peak/peak $H R: 0.2 \pm 0.06$ and

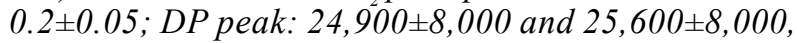
and $\%$ peak HR/predicted HRmax: $91.3 \pm 9$ and $91.1 \pm 11$. The following values were significant: preobservation $\mathrm{VO}_{2}$ peak versus postobservation $\mathrm{VO}_{2}$ peak in $\mathrm{GI}(\mathrm{p}=0.0$ $063)$; postobservation $\mathrm{VO}_{2}$ peak in $\mathrm{GI}$ versus postobservation $\mathrm{VO}_{2}$ peak in $\mathrm{GII}(\mathrm{p}=0.0045)$; postobservation $\mathrm{VO}_{2}$ peak/peak $\mathrm{HR}$ GI versus postobservation peak $\mathrm{VO}_{2} /$ peak HR in GII ( $p=0.0000)$. The follow-up periods in GI and GII were, respectively, $41.33 \pm 20.19$ months and 20.60 18.16 months $(p<0.05)$. No difference between the groups was observed in coronary risk factors, therapeutic management, or evolution of ischemia. No cardiovascular events secondary to USR were observed in 620 patient-months.

Conclusion - USR was safe and efficient, in low-risk patients with coronary artery disease and provided benefits at the peripheral level.

Key words: atherosclerotic coronary artery disease, exercise test, unsupervised rehabilitation

Universidade Federal de São Paulo - Escola Paulista de Medicina

Mailing address: Japy Angelini Oliveira Filho - Rua Tapejara, 109 - 05594-050

São Paulo, SP, Brazil - E-mail: japyoliveira@uol.com.br

English version by Stela Maris C. e Gandour
Cardiac rehabilitation is currently a routine procedure for the treatment of atherosclerotic coronary artery disease. It may be performed at specialized centers (supervised rehabilitation), at home, or in public places with long-range supervision (unsupervised rehabilitation) ${ }^{1}$. Unfortunately, supervised rehabilitation has benefited few patients, even in developed countries, due both to logistic difficulties and high operational costs ${ }^{2}$. The major objective of unsupervised rehabilitation is for patients to exercise under indirect supervision, extending the practice of exercising to a large number of patients considered low risk for ischemia when practicing physical exercise ${ }^{3}$. In this context, unsupervised rehabilitation has been considered a safe activity ${ }^{4}$.

Studies reported in the medical literature on unsupervised rehabilitation are few. The first guidelines for unsupervised rehabilitation were published by Williams et al in $1981^{5}$. Since the first study ${ }^{6}$ until the present date, 13 original articles and a summary have been reported, adding to a total of 1,230 patients with coronary artery disease exercising according to protocols from 4 to 24 weeks after an acute coronary event ${ }^{7-18}$. In Brazil, only recently have the guidelines and the first experiences on unsupervised rehabilitation been published ${ }^{19-21}$.

Patients with antecedents of coronary artery disease spontaneously restrict their physical activity, independent of medical guidance. This defensive behavior interferes with work, recreation, and personal activities, sexual relationships included. Several studies have shown the importance of physical training in these patients with improvement in health status and psychological profile $\mathrm{e}^{1-4,19}$.

The objectives of this study were as follows: 1 ) to assess the performance of patients with stable atherosclerotic coronary artery disease at low risk for developing ischemia during exercise practice ${ }^{5}$ in the long run using a protocol of unsupervised rehabilitation; and 2) to assess efficacy, safety, and feasibility of unsupervised rehabilitation conducted from the medical office in our environment. 


\section{Methods}

The design of the protocol was that of a case-control study ${ }^{22}$. In a retrospective analysis of medical records from a private practice, we consecutively selected 20 patients with stable atherosclerotic coronary artery disease confirmed on coronary angiography who were considered at low risk for developing ischemia during exercise according to the modified criteria of Williams et al ${ }^{5}$ (chart I: criteria 2,3, and 4). These patients underwent a regular program of unsupervised rehabilitation during a period longer than 12 months between 1982 and 1996. From this group, 15 patients were selected to undergo unsupervised rehabilitation and constituted group I(GI). Four patients were excluded due to insufficient information in their medical records, and another patient was excluded due to the impossibility of obtaining parity in the control group. GI was compared with the control group (GII), which comprised 15 patients selected from the outpatient clinic for coronary artery disease of the cardiology department of the Escola Paulista de Medicina (UNIFESP-EPM). These GII patients were selected in a retrospective assessment of medical records and were paired by age, sex, clinical findings, and ambulatory follow-up longer than 12 months in the same period. Each group had 13 males and 2 females with angina pectoris $(n=8)$, previous myocardial infarction $(\mathrm{n}=8)$, antecedents of coronary angioplasty $(n=2)$, and revascularization surgery $(n=6)$. Ages of GI and GII patients were $64 \pm 10$ and $63 \pm 10$ years, respectively. The distribution of the major coronary risk factors was similar in both groups as follows (GI and GII, respectively): smoking ( 6 vs 5 cases), arterial hypertension ( 9 vs 11 cases), hypercholesterolemia ( 9 vs 10 cases), and diabetes mellitus (4 vs 5 cases).

According to the protocol, GI patients were informed about the risks and benefits of physical training. Initially, all patients underwent clinical and laboratory assessments to

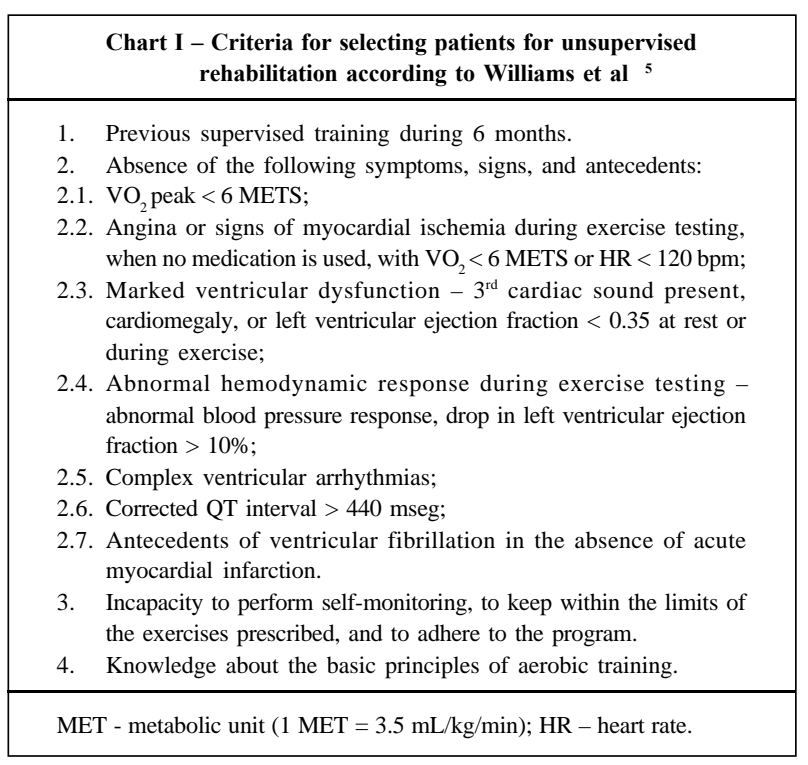

stratify the exercise risk ${ }^{5}$. Then, they were motivated to exercise according to their own preferences and availabilities after an extensive explanation about the fundamentals of physical conditioning. The patients were periodically reassessed on an outpatient basis and were advised to suspend exercise practice and to look for medical assistance in case of incidents. The exercise program comprised 3 to 5 sessions per week, lasting 30 to 60 minutes each and reaching $70 \%$ to $80 \%$ of the useful functional capacity (maximal exercise level in the absence of symptoms or clinical or electrocardiographic signs) ${ }^{23}$. Physical training comprised jogging $(n=12)$, exercising on a treadmill $(n=2)$, swimming $(n=2)$, tennis $(n=1)$, and soccer $(n=1)$. The patients who chose swimming and tennis were used to those sports modalities. The patient who chose soccer despite the warning that that modality of exercise was inadequate and even risky kept that practice on a regular basis ${ }^{24}$.

GII patients were routinely followed up in the outpatient clinic with usual hygienic and dietary measures and clinical treatment.

At the beginning and end of the observation period, GI and GII patients underwent conventional exercise testing using their usual medications. On the initial assessment, the following protocols were used: 1 ) in GI-Bruce ( $n=5)$, classic Ellestad $(\mathrm{n}=5)$, attenuated Ellestad $(\mathrm{n}=1)$, and Astrand $(\mathrm{n}=4) ; 2)$ in GII-Bruce $(\mathrm{n}=12)$ and modified Naughton $(\mathrm{n}=3)$. On the final assessment, the following protocols were used: 1) in GI - Bruce ( $n=5)$, classic Ellestad $(n=8)$, and Astrand $(\mathrm{n}=2) ; 2)$ in GII - Bruce $(\mathrm{n}=15) . \mathrm{VO}_{2}$ peak was estimated according to the formulae and nomograms of the protocols used and the reports of the tests. For standardization, the exercise tests were reassessed according to the criteria established by the Consenso Nacional de Ergometria ${ }^{25}$. The estimations of $\mathrm{VO}_{2}$ peak were then recalculated according to the recent standardization ${ }^{26}$ for the Bruce ${ }^{27}$, Ellestad ${ }^{28}$ and Naughton ${ }^{29}$ tests, and correction factors were applied for the cycloergometric tests ${ }^{30}$.

The statistical analysis was performed with the Student $t$ test ( 2 -tailed), and the values of $\mathrm{p}<0.05$ were considered significant.

\section{Results}

The observation periods for GI and GII patients were $41.3 \pm 20.2$ and $20.6 \pm 8.2$ months, respectively ( $p<0.05)$. GI patients were followed up for a variable period of 19 to 79 months (median of 31 months), which comprised 620 patient-months of observation with no cardiovascular accidents secondary to the program.

Figures 1,2,3, and 4 summarize the results using $\mathrm{VO}_{2}$ peak values obtained in the medical records. In GI, the following pre-and postobservation values were respectively obtained: $\mathrm{VO}_{2}$ peak (mL/kg/min): $24 \pm 5$ and $31 \pm 9 ; \mathrm{VO}_{2}$ peak $/ \mathrm{HR}$ peak: $0.18 \pm 0.05$ and $0,28 \pm 0,13$; DP peak: $26,800 \pm 7,000$ and $29,000 \pm 6,500$; and $\% H R$ peak/predicted HRmax: $89.5 \pm 9$ and $89.3 \pm 9$. In GII, the following pre- and postobservation values were respectively obtained: $\mathrm{VO}_{2}$ peak $(\mathrm{mL} / \mathrm{kg} / \mathrm{min})$ : 


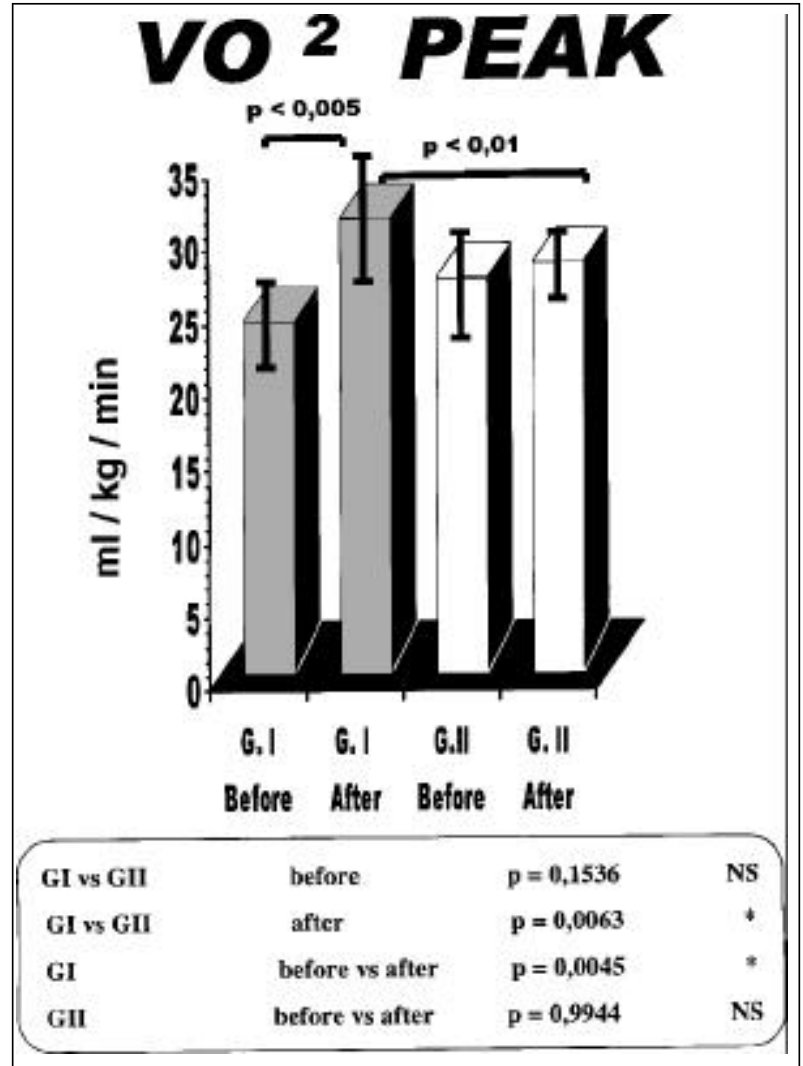

Fig. 1 -Peak $\mathrm{VO}_{2}$ before and after follow-up in groups I (experimental) and II (paired control).

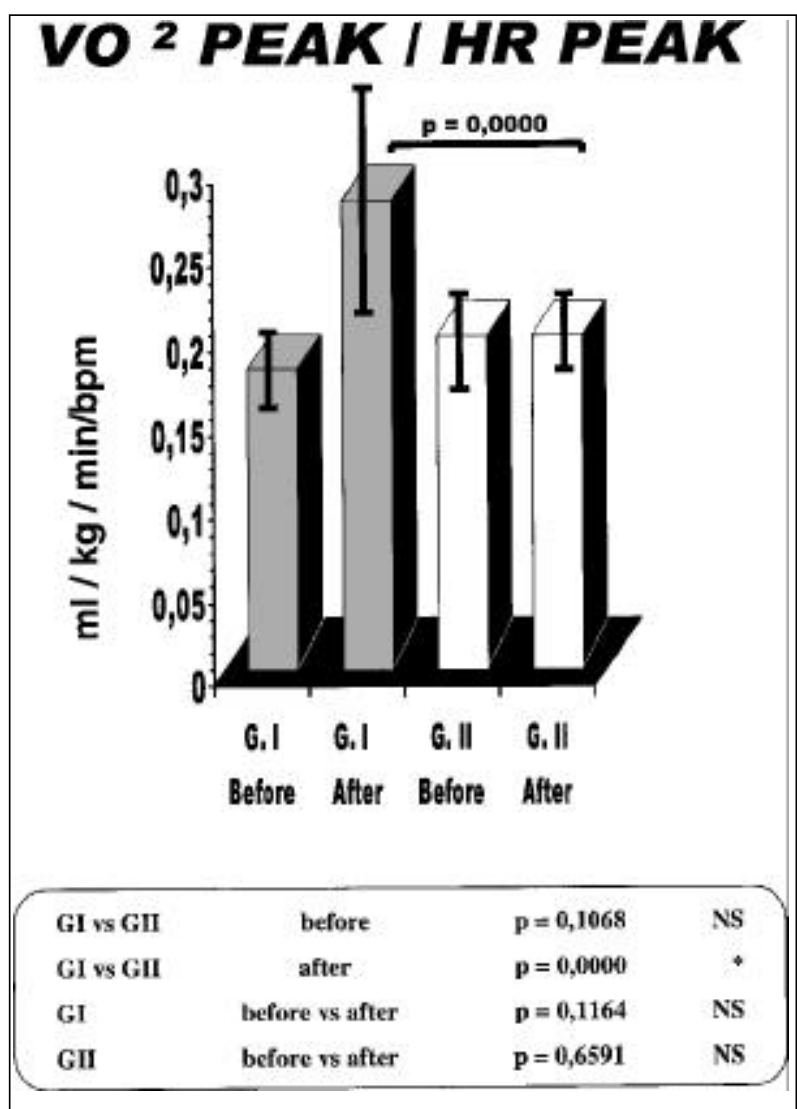

Fig. 2 - Peak $\mathrm{VO}_{2}$ /peak $\mathrm{HR}$ ratio before and after follow-up in groups I (experimental) and II (paired control).



Fig. 3 - Peak double product before and after follow-up in groups I (experimental) and II (paired control).

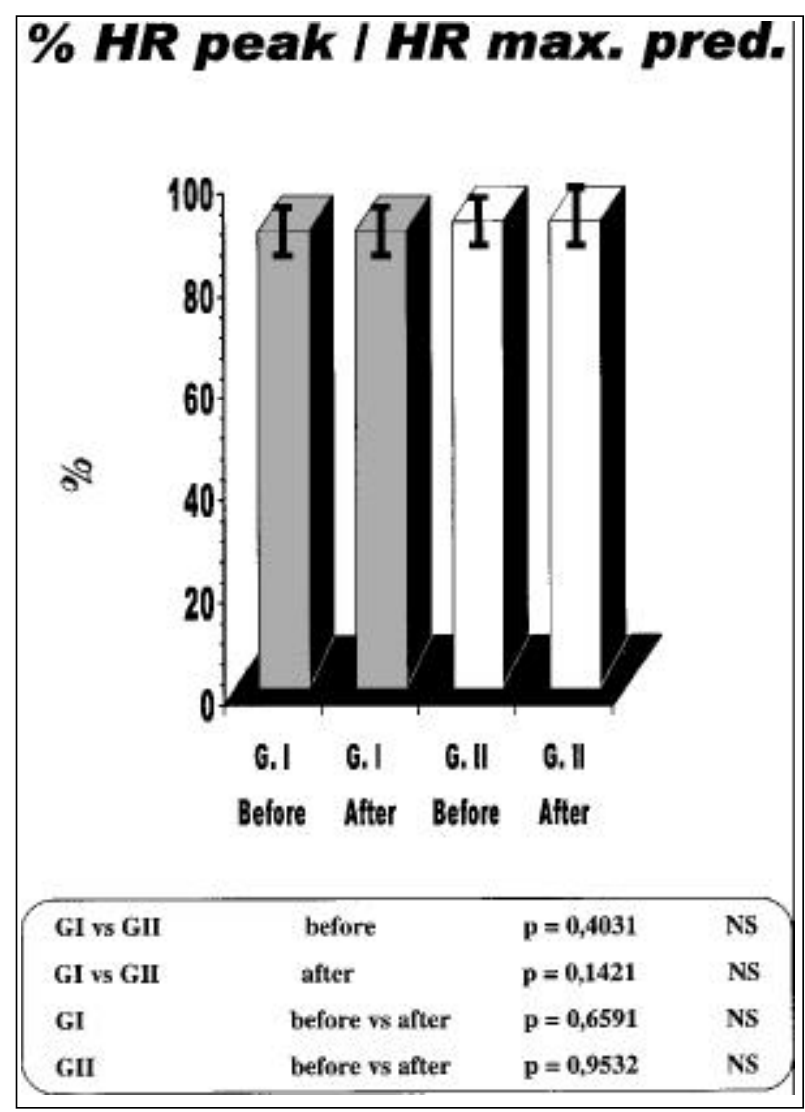

Fig. 4 - Peak HR/predict maximal HR*(\%) before and after follow-up in groups I (experimental) and II (paired control). * Predict maximal HR $=220$ - age (in years). 
$27 \pm 7$ and $28 \pm 5 ; \mathrm{VO}_{2}$ peak/HR peak $0.2 \pm 0.06$ and $0.2 \pm 0.05 ; \mathrm{DP}$ peak: $24,900 \pm 8,000$ and $25,600 \pm 8,000$; and $\%$ HR peak/predicted HRmax: $91.3 \pm 9$ and $91.1 \pm 11$.

The statistical analysis showed the following statistically significant differences: pre- $\mathrm{VO}_{2}$ peak versus post- $\mathrm{VO}_{2}$ peak in $\mathrm{GI}(\mathrm{p}=0.006)$; post- $\mathrm{VO}_{2}$ peak in GI versus post- $\mathrm{VO}_{2}$ peak in GII $(\mathrm{p}=0.004)$, and post- $\mathrm{VO}_{2}$ peak/HR peak in GI versus post- $\mathrm{VO}_{2}$ peak/HR peak in $\mathrm{GII}(\mathrm{p}=0.0001)$.

The evolution of the myocardial ischemia pattern during exercise testing was similar in GI and GII (tab. I). No myocardial revascularization was performed in either group during the observation period.

\section{Discussion}

The results of this study are the $1^{\text {st }}$ published about unsupervised rehabilitation in the long run in the specialized literature ${ }^{20,21}$.

Currently, only $25 \%$ of the patients with coronary artery diseases are estimated to have access to clinics for supervised rehabilitation ${ }^{2}$. Logistic, financial, and social difficulties hinder the wide use of supervised rehabilitation in that population. In our environment, clinics specializing in supervised rehabilitation are lacking. In that context, unsupervised rehabilitation allows patients considered at low risk for ischemic events to exercise in public places and even in their homes. In reality, the term "unsupervised rehabilitation," which has been widely spread, is not appropriate, because the patient is required to maintain periodic contact with the physician. Even though multidisciplinary teams have been advised to work in unsupervised rehabilitation, it is the assistant physician who accounts for the patient's admission, prescription, assessment of clinical repercussions, and discharge from the training program ${ }^{31}$. In our opinion, the term "semi-supervised rehabilitation" would be more appropriate $^{32}$.

In the present study, patients were advised about the exercise program during their usual medical visits. This proved feasible, and, considering the importance of the physician-patient relationship, this could be an advantage for the better acceptance of the program. Several guidelines on unsupervised rehabilitation have been published ${ }^{5,19,32-35}$. According to most of these guidelines, unsupervised rehabilitation should start with a brief period of training in spe-

Table I - Evolution of the pattern of myocardial ischemia during exercise testing in pre- and postobservation GI and GII

\begin{tabular}{|c|c|c|}
\hline Evolution & Group I & Group II \\
\hline pre ET - to post ET - & 7 cases & 10 cases \\
\hline pre $\mathrm{ET}+$ to post $\mathrm{ET}+$ & 2 cases & 3 cases \\
\hline pre ET - to post ET + & 3 cases & 1 cases \\
\hline pre $\mathrm{ET}+$ to post ET - & 3 cases & 1 cases \\
\hline \multicolumn{3}{|c|}{$\begin{array}{l}\text { pre ET - preobservation nonischemic exercise test; pre ET+ } \\
\text { preobservation ischemic exercise test; post ET- postobservation } \\
\text { nonischemic exercise test; post ET+ - postobservation ischemic } \\
\text { exercise test. }\end{array}$} \\
\hline
\end{tabular}

cialized clinics. As happened in our study, in several cases that period can be replaced by the delivery of speeches and theoretical-practical instructions.

In our study initiated in 1982, the criteria for patient selection proposed by Williams et al ${ }^{5}$ were used. It is worth noting that unsupervised rehabilitation is appropriate for low-risk patients with coronary artery diseases. The criteria for defining low-risk patients are very similar, except for some small differences ${ }^{4,5,19}$. The patients in our study were in a stable, chronic phase and had not undergone a resting period due to a recent coronary event. Despite this, the mean increase in $\mathrm{VO}_{2}$ peak reached $29 \%$ during training longer than 12 months, confirming the benefit of exercise in the long run. After physical training, $\mathrm{VO}_{2}$ peak $(\mathrm{mL} / \mathrm{kg} / \mathrm{min})$ increased from $24 \pm 5$ to $31 \pm 9$ in GI. These data correspond to the mean elevation of 2 METS, which can significantly increase the patient's functional class and interfere with work, recreation, and personal activities, sexual relationships included ${ }^{36}$. In the initial phase, both groups had similar $\mathrm{VO}_{2}$ peak values. In GII, no significant variation was observed in $\mathrm{VO}_{2}$ peak, confirming that the final values recorded in GI were due to physical training.

Because this was a retrospective study, uniformity in the protocols of the exercise test was not possible. However, Bruce and Ellestad protocols were used in $85 \%$ of the exercise tests. Those protocols had a high correlation in the regression analyses, and no significant differences were observed in the measurements of $\mathrm{VO}_{2}$ peak, peak heart rate, and peak blood pressure ${ }^{37}$. In $10 \%$ of the exercise tests, the Astrand protocol for the cycloergometer was used. The mean $\mathrm{VO}_{2}$ peak on a bicycle ranged from $89 \%$ to $95 \%$ of the values found on treadmills, and a $10 \%$ increase in the values obtained with the cycloergometer was considered corresponding to the values obtained on the treadmill ${ }^{30}$. In this way, all calculations and statistical analyses referring to the $\mathrm{VO}_{2}$ peak estimates were redone, this correction included ${ }^{30}$, according to recent standardization ${ }^{26}$. Nevertheless, the statistical analysis also showed similar results.

Our results are in accordance with those in the literature ${ }^{6-18}$ that report a greater aerobic power in the groups trained as compared with that in untrained groups. However, unlike our sample, which comprised patients selected who were in a stable, chronic phase, other studies lack values of preconditioning aerobic power, because they were started after recovery from an acute coronary event. Usually, 23\% elevations of $\mathrm{VO}_{2}$ peak are reported during a conditioning period ranging from 1 to 6 months ${ }^{6,8,9,10,12,17}$, as is a $19 \%$ elevation of the mean anaerobic threshold ${ }^{8,10,11}$. After 4 weeks of training, significant elevations in the maximal aerobic power by $14 \%$ and in the 2 mmol lactate threshold by $19 \%$ were detected ${ }^{18}$.

Maximal heart rate obtained during exercise testing may be predicted using equations and serve to assess whether the effort was sufficient. Usually in patients with coronary artery disease, maximal heart rate is assessed using the heart rate measured at the peak of exertion, which is called peak heart rate. Maximal heart rate decreases with 
age. Therefore, for a long-term assessment of patients of different ages, the percentage reached by the peak heart rate is more appropriate, because it is a linear function of the exercisedpercentageof $\mathrm{VO}_{2}$ peak $^{38}$. In GI and GII, the percentages of the predicted maximal heart rate reached during the tests were $89 \%$ and $91 \%$, respectively ( $p>0.05)$. In this way, possible biases of $\mathrm{VO}_{2}$ peak measurement were excluded and the degrees of quality of the exercise tests performed were validated.

The $\mathrm{VO}_{2}$ peak/HR peak relation had not been analyzed in previous publications. In our study, a significant elevation of the pre- and postconditioning $\mathrm{VO}_{2}$ peak/HR peak relation occurred in GI, showing a greater efficacy of myocardial work and confirming the benefit of exercising in the long run. However, maintenance of double product levels, which usually render myocardial $\mathrm{O}_{2}$ consumption, reflects a predominantly peripheral response to physical exercise.

For unsupervised rehabilitation, data in the literature are controversial regarding the repercussions of training on ventricular function. After 6 months of exercising, a mild elevation in ejection fraction at rest has been reported only in patients with reduced ventricular function ${ }^{11}$. In a protocol for physically disabled individuals, significant elevations in ejection fraction and percentage of systolic shortening occurred at peak exertion, maintaining the values at rest unaltered ${ }^{14}$. However, elevations of the systolic volume at rest and during exercise, with no alteration in the ejection fraction, have been reported in patients undergoing highintensity exercising; these values remained unaltered in the subgroup of patients exercising at low intensity ${ }^{17}$.

In our study, the pattern of evolution of myocardial ischemia observed during exercise testing showed no significant alterations, being similar in both groups. In a pioneering study, Miller et $\mathrm{al}^{6}$ reported ischemic tests in $42 \%$ and $35 \%$ of the patients at the beginning and end of the protocol, respectively.
No conclusive data about the risk of unsupervised rehabilitation exist. As this was a retrospective study, we were not able to assess the patients' compliance with the exercise program based on the medical records. However, no accident occurred in 620 patient-months of observation.

The compliance reported by other authors has been satisfactory in prospective protocols of unsupervised rehabilitation. In the literature, compliances of $89 \%$ and $72 \%$, respectively, have been reported after up to 11 and 26 weeks of conditioning ${ }^{6}$ and of $91 \%$ and $83 \%$, respectively, by the end of 4 and 24 weeks of training ${ }^{9}$. Considering an eventual compliance of $70 \%$ and an average training of 3 sessions per week, no accidents would have been reported in approximately 5,200 patient-hours of training.

The risk of cardiocirculatory arrest in unsupervised rehabilitation was $1 / 6,000$ patient-hours in the 1970 s, decreasing to $1 / 120,000$ in the 1990 s. Recently, risks of cardiocirculatory arrest of $1 / 98,717$ patient-hours (supervised rehabilitation) and of 1/70,000 patient-hours (unsupervised rehabilitation) have been reported ${ }^{4}$. According to these findings, approximately 1 cardiocirculatory arrest occurred for each group of 100 patients exercising uninterruptedly for 5 years, in a regimen of 3 sessions per week. The availability of emergency devices in public places used for unsupervised rehabilitation can be extremely useful ${ }^{39,40}$. In our relatively small sample comprising 5,200 patient-hours of training, no coronary event related to training occurred, which is in accordance with reports in the specialized literature.

Considering the limitations of ours, new prospective studies are required with larger samples subdivided into specific groups of patients with atherosclerotic coronary artery disease. These results may be partially due to the enthusiasm for exercise in the selected patients. Subsequent studies using proper questionnaires may confirm an improvement in quality of life.

\section{References}

1. Balady GJ, Ades PA, Comoss P, et al. Core components of cardiac rehabilitation/ secondary prevention programs. Circulation 2000; 102: 1069-73.

2. Franklin BA, Hall L, Timmis GC. Contemporary cardiac rehabilitation services Am J Cardiol 1997; 78: 1075-7.

3. De Busk RF. Treinamento por exercício em residência e no local para doença coronariana. Clínicas Cardiológicas 1993; 2: 295-305.

4. Fletcher GF, Balady G, Froelicher VF, et al. ACC/AHA Special report: exercise standards. Circulation 1995; 82: 2286-322.

5. Williams RS, Miller H, Koisch P, et al. Guidelines for unsupervised exercise in patients with ischemic heart disease. J Card Rehab 1981; 1: 213-9.

6. Miller NH, Haskell WL, Berra K, De Busk RF. Home versus group exercise training for increasing functional capacity after myocardial infarction. Circulation 1984; 70: 645-9.

7. Kugler J, Dimsdale JE, Hartley H, Sherwood J. Hospital supervised vs home exercise in cardiac rehabilitation: effects on aerobic fitness, anxiety, and depression. Arch Phys Med Rehabil 1990; 71: 322-5.

8. Ueshima K, Saito M, Shimohara A, et al. Management and evaluation of nonsupervised home exercise program in convalescent phase of acute myocardial infarction. Jap Circulation J 1990; 54: 1437-40.

9. Thomas RJ, Miller NH, Taylor CB, Ghandour G, Fisher L, DeBusk RF. Nursemanaged Home-Based exercise training after acute myocardial infarction: methods and effects on functional capacity. Circulation 199; 84(Suppl II): 540.
10. Shimohara A, Ueshima K, Saito M, et al. Non-supervised home exercise programs in a convalescent phase of acute myocardial infarction: their effectiveness and usefulness of the heart rate at anaerobic threshold. J Cardiol 1991; 21;309-15.

11. Giannuzzi p, Tavazzi L, Temporelli Pl, et al. Long-term physical training and left Ventricular remodeling after anterior myocardial infarction: results of the exercise in EAMI trial. J Am Coll Cardiol 1993; 22: 1821-9.

12. Aros F, Armentia J, Castillo C, Cordo JC, Loma-Osorio A. Evaluation del entrenamiento físico domiciliario precoz despus de un infarto agudo de miocardio. Rev Esp Cardiol 1993; 46: 544-41.

13. Sparks KE, Shaw DK, Eddy, D Hanigosky P, Vantrese J. Alternatives for cardiac rehabilitation patients unable to return to a hospital-based program. Heart \& Lung 1993; 22: 298-303.

14. Fletcher BJ, Dunbar SB, Felner JM, et al. Exercise testing and training in physically disabled men with clinical evidence of coronary artery disease. Am J Cardiol 1994; 73: 170-4.

15. Froelicher ES, Kee LL, Newton KM, Lindskog B, Livingston M. Return to work, sexual activity and other activities after acute myocardial infarction. Heart Lung 1994; 23: 423-35.

16. Brosseau R, Juneau M, Sirard A, et al. Safety and feasibility of a self-monitored, home-based phase II exercise program for high-risk patients after cardiac surgery. Can J Cardiol 1995; 11: 675-85. 
17. Adachi H, Koike A, Obayashi T, et al. Appropriate endurance exercise training improve cardiac function in patients with prior myocardial infarction? Europ Heart J 1996; 17: 1511-21.

18. Bjarnason-Wehrens B, Predel HG, Graf C, Gunter D, Rost R. Improvement of physical performance and aerobic capacity mediated by a novel 4-week ambulatory cardiac rehabilitation program. Z Cardiol 1999; 88: 113-22.

19. Godoy M, Bellini AJ, Pássaro LC, et al. SBC/DERC I Consenso Nacional de Reabilitação Cardiovascular. Arq Bras Cardiol 1997; 69: 267-92.

20. Oliveira FJA, Leal AC, Salles AF, Mesquita F, Leite W, Santos FDV. Reabilitação não supervisionada em consultório. Arq Bras Cardiol 1999; 73 (suppl 6): 57.

21. Oliveira FJA, Leal AC, Lima VC, Santos FDV, Luna FB. Rev Soc Cardiol Estado de São Paulo 2000; 10(supl B): 49.

22. Schlesselman JJ. Case control studies: design, conduct and analyse. Oxford University Press: New York, 1982: 7-26.

23. Boskis B, Lerman J, Perósio AMA, Scattini MC. Manual de Ergometria y rehabilitation en Cardiologia. Cordoba: ECTA,1974: 160-1.

24. $26^{\text {th }}$ Bethesda conference. Recommendations for determining eligibility for competition in atlhetes with cardiovascular abnormalities ACSM/ACC. Med Sci Sport Exer 1994; 26(suppl): 223-83.

25. Mastrocolla LE, Brito AX, Brito FS, et al. SBC/DERC Consenso Nacional de Ergometria. Arq Bras Cardiol 1995; 65: 189-210.

26. Fardy PS, Yanowitz FG, Wilson PK. Reabilitação cardiovascular. Rio de Janeiro: Revinter, 2000: 106

27. Bruce RA, Kusumi F, Hosmer D. Maximal oxigen intake and nomographic assessment of functional impairment in cardiovascular disease. Am Heart J 1973; 85: 346-51.

28. Ellestad MH, Allen WA, Wan MLK, Kemp GL. Maximal treadmill stress testing for cardiovascular evaluation. Circulation 1969; 39: 517-23.
29. Naughton J, Balke B, Sevelius G. Physiological responses of normal and pathological subjects to a modified work capacity test. J Sports Med 1963; 3: 201-07.

30. Wasserman K, Hansen JE, Sue DY, Whipp BJ, Casaburi R. Principles of exercise testing and interpretation. $2^{\text {nd }}$ ed. Philadelphia: Lea \& Febiger, 1994

31. Conselho Federal de Medicina. Resolução 1236/87, 1987.

32. Oliveira FJA, Salvetti XM. Programas não supervisionados em reabilitação cardiovascular. Abordagem da prescrição dos exercícios. Rev Soc Cardiol Estado de São Paulo 1996; 6: 31-9.

33. Oliveira FJA. Cardiopatia isquêmica e exercício. In: Ramos OL, Rothshield H. Atualização Terapêutica. São Paulo: Artes Médicas, 1999.

34. Yazbek Jr P, Santomauro AC, Wajngarten M, Azul LGS, Battistella LR, Pilleggi F. Exercício não supervisionado para cardiopatas: imperativos. Arq Bras Cardiol 1993; 60: 51-2.

35. Quittan M. Ambulante medizinische trainingstherapie bei koronarer herzkrankheit. Wien Med Wschr 1993; 143: 20-6

36. Stein RA. The effect of exercise training on heart rate during coitus in the post myocardial infarction patients. Circulation 1977; 55: 738-40.

37. Pollock ML, Bohannon RL, Cooper KH, et al. A comparative analyses of four protocols for maximal treadmill stress testing. Am Heart J 1976; 92: 39-46.

38. Haskell WL. Design and implementation of cardiac conditionning programs. In: Wenger NK, Hellerstein HK. Eds. Rehabilitation of Coronary Patient. New York: John Wiley \& Sons, 1978.

39. Rost RA. Atividade física e o coração. Rio de Janeiro: MEDSI, 1991: 110

40. Oliveira FJA. Reabilitação cardíaca em consultório. Âmbito Med Desportiva $1995 ; 1: 13-20$ 